\title{
High-resolution microscopy of plasmon field distributions by scanning tunneling luminescence and photoemission electron microscopies
}

\author{
Microscopie à haute résolution des champs plasmoniques par microscopie tunnel \\ à balayage de sonde et par microscopie de photoémission multiphotonique
}

\author{
Ludovic Douillard, Fabrice Charra* \\ CEA-Saclay, service de physique et chimie des surfaces et interfaces, IRAMIS, 91191 Gif-sur-Yvette cedex, France
}

\section{A R T I C L E I N F O}

\section{Article history:}

Available online 6 November 2012

\section{Keywords:}

STM

PEEM

Subnanometer spatial resolution

\section{Mots-clés:}

Microscopie tunnel à balayage de sonde

Imagerie de photoémission multiphotonique

Résolution spatiale inférieure au nanomètre

\begin{abstract}
A B S T R A C T
The exploitation of plasmon resonances to promote the interaction between conjugated molecules and optical fields motivates intensive research. The objectives are to understand the mechanisms of plasmon-mediated interactions, and to realize molecularly- or atomically-precise metal nanostructures, combining field enhancements and optical antenna effects. In this review paper, we present examples of plasmonic-field mappings based on scanning tunneling microscope (STM)-induced light emission or multiphoton photoemission (PEEM), two techniques among those which offer today's best spatial resolutions for plasmon microscopy. An unfamiliar property of the junction of an STM is its ability to behave as a highly localized source of light. It can be exploited to probe optoelectronic properties, in particular plasmonic fields, with ultimate subnanometer spatial resolution, an advantage balanced by a sometimes delicate deconvolution of local-probe influence. Alternatively, local-probe disadvantages can be overcome by imaging the photoemitted electrons, using well-established electron optics. This allows obtaining two-dimensional intensity maps reflecting the unperturbed distribution of the optical near field. This approach provides full field spectroscopic images with a routine spatial resolution of the order of $20 \mathrm{~nm}$ (down to $5 \mathrm{~nm}$ with recent aberration corrected instruments).
\end{abstract}

(C) 2012 Académie des sciences. Published by Elsevier Masson SAS. All rights reserved.

\section{R É S U M É}

L'exploitation des résonances plasmons dans le but de promouvoir l'interaction entre des molécules conjuguées et des champs optiques motive actuellement d'intenses recherches. Les objectifs en sont la compréhension du rôle médiateur des interactions optiques joué par les modes de plasmon et la réalisation de nanostructures métalliques avec une précision moléculaire voire atomique, combinant les effets d'exaltation de champ et d'antennes optiques. Dans cet article de synthèse, nous présentons des exemples de cartographie des champs plasmoniques basés sur deux techniques de microscopie : l'émission de lumière induite par microscopie tunnel à balayage de sonde (scanning tunneling microscopy STM) et l'imagerie de photoémission multiphotonique (photoemission electron microscopy - PEEM), deux techniques parmi celles qui offrent aujourd'hui les meilleures résolutions spatiales pour la microscopie plasmonique. Une propriété peu conventionnelle de la jonction du microscope à effet tunnel est sa capacité à se comporter comme une source localisée de lumière. Celle-ci peut être exploitée pour sonder localement les propriétés

\footnotetext{
* Corresponding author.

E-mail address: fabrice.charra@cea.fr (F. Charra).
} 
opto-électroniques en surface, en particulier les modes plasmoniques, avec une résolution spatiale inférieure au nanomètre. L'avantage de cette résolution ultime est cependant contrebalancé par la nécessité d'une déconvolution parfois délicate de l'influence de la sonde. Alternativement, les inconvénients inhérents aux techniques de sondes locales peuvent être surmontés par l'imagerie des électrons photoémis, en utilisant les méthodes bien établies d'optique électronique. Ceci permet l'obtention de cartes d'intensité en deux dimensions reflétant directement la distribution non perturbée du champ proche optique. Cette approche fournit des images avec une résolution spatiale de l'ordre de $20 \mathrm{~nm}$ en routine et pouvant atteindre $5 \mathrm{~nm}$ avec les instruments les plus récents, incluant un dispositif de correction des aberrations.

(c) 2012 Académie des sciences. Published by Elsevier Masson SAS. All rights reserved.

\section{Introduction}

Plasmon oscillation modes are eigenmodes of the combined electromagnetic fields and collective electron oscillations. In the close vicinity of noble-metal nanostructures and at specific frequencies in the optical range, plasmon modes are at the origin of strong local enhancements of the near-field optical intensity, with enhancement factors as large as several orders of magnitudes compared to the corresponding in- or out-coupled plane wave at the same frequency. A well-known consequence is the appearance of increased optical absorptions forming narrow peaks in the absorption spectra at plasmon resonance frequencies, as first evidenced by Faraday in the nineteenth century [1]. This effect has for consequence an increased electromagnetic coupling between quantum systems located in their vicinity and optical fields. This spatialdomain variant of the Purcell effect which involves frequency-domain optical density of states [2], thus promotes energy exchanges between quantum system and optical fields. In order to yield observable consequences in the far field, those enhancements of local interactions must be combined with optical antenna effects allowing their coupling with propagative optical waves. The amplification role played by local plasmon modes is now clearly established in many photonic phenomena such as surface-enhanced Raman scattering (SERS) at rough noble-metal surfaces [3], inelastic fast-electron scattering by clusters of metal nanoparticles [4], amplification of nonlinear optical properties [5] or anomalously high light transmission through sub-wavelength apertures in noble-metal films [6]. Plasmonics, that is, the ensemble of techniques exploiting the specific properties of plasmons in optoelectronic and photonic devices, currently constitutes a very active field of applied research. The main goals are to understand the mechanisms of plasmon-mediated interactions in order to be able to realize molecularly- or atomically-precise metal nanostructures with specific plasmonic responses.

Progress in this domain requires a deep understanding of the subtle interplay between field enhancements and antenna effects. To this aim, a key challenge is the access to experimental tools allowing for the investigation of the optical nearfield spatial distribution at the nanometer scale. To date this issue is commonly addressed using scanning near-field optical microscopy (SNOM) or related methods $[7,8]$. These techniques offer routine spatial resolutions down to several tens of nanometers $(50-100 \mathrm{~nm})$.

Although below the diffraction barrier, such a resolution is often still not enough to resolve the highly confined hot spots which are responsible for largest enhancements. This motivates efforts to develop alternative techniques based on so-called active probes, i.e. embedding their own source of light. From this point of view, the ability of the nanoscale junction of an STM to behave as a highly localized source of light may be exploited. For biases of $\sim 1 \mathrm{~V}$ and above this source has frequencies in the optical domain. It can thus probe optoelectronic properties with subnanometer spatial resolution. The cost of this unparalleled resolution is the need for deconvolution of local-probe influence, which is even more delicate here than in the case of standard passive local-probe techniques, since the properties of the active probe may vary while scanning an inhomogeneous sample.

To get rid of local-probe perturbations in near-field mapping, the possibly strong enhancements of electron photoemission upon excitation of surface plasmons can be cunningly exploited. By collecting the photoemitted electrons, twodimensional intensity maps reflecting the actual distribution of the optical near field are obtained. The imaging technique makes use of well-established electron optics, i.e. involves no physical probe altering the measure. This approach provides full field spectroscopic images with a routine spatial resolution of the order of $20 \mathrm{~nm}$ reaching down to $5 \mathrm{~nm}$ with recent aberration corrected instruments [9].

In this review paper, we present examples of plasmonic-field mappings based on STM-induced light emission or photoemission microscopy, two techniques which offer today's best spatial resolutions for plasmon microscopy.

\section{Investigation of plasmon modes, from excitation to damping}

Surface plasmons (SP) have been investigated either from the excitation side or the damping side of the radiation-matter interaction. Considering first the excitation ways, surface plasmons can be excited by (i) electromagnetic radiations, that is visible or near infrared light. However, the SP dispersion curve lies entirely to the large-vave vector side of the light line and the fulfillment of momentum conservation at a given photon frequency implies an increase of the light wave vector. Two methods are widely used, namely the grating coupler [10] or the attenuated total internal reflection (ATR) technique [11,12]; 
(ii) excitation by fast electrons. Upon scattering, electrons transfer momentum and energy to the solid. The component of the scattering vector parallel to the interface determines the SP wave vector and, together with the dispersion relation, the energy loss of the scattered particles [13].

The damping processes of a surface plasmon constitute also access ways to the investigation of plasmon physics. Symmetrically to the light excitation process, a surface plasmon can (iii) radiate its energy back into free space. As for the excitation scheme, this radiation damping implies condition on the momentum conservation law. Random roughness and/or specifically designed diffraction gratings are common additional momentum suppliers. Another important damping channel corresponds to (iv) internal absorption through ohmic losses. In short, a plasmon can decay non-radiatively by excitation of single (electron, hole) pairs, whose further coupling with phonons translates into heating. (v) Electron emission through either linear or nonlinear photoelectric effects can also be invoked for SP energy relaxation. Finally (vi) coupling to secondary surface plasmons polaritons (SPPs) or localized surface plasmons (LSPs) offers additional relaxation paths [14].

These excitation/deexcitation schemes have paved the way for the development of investigation tools in plasmonics. Indeed, surface plasmons can be observed using far-field optical techniques taking advantage of the scattering due to residual roughness. Far-field methods, including nonlinear techniques such as two-photon photoluminescence, are diffraction limited and thus of only limited use at length scales below $200 \mathrm{~nm}$ [15]. Near-field techniques such as photon scanning tunneling microscopy (PSTM) [16], scanning near-field optical microscopy (SNOM) [17] and apertureless SNOM [18] achieve better spatial resolution down to sub-wavelength dimensions (50-100 nm). However, these scanning probe techniques require physical probes whose reproducibility continues to be a true experimental challenge and may suffer from probe-sample interactions distorting the signal of interest [13].

Plasmon excitation by fast electrons has given birth to efficient near-field mapping methods, such as electron energy loss spectrometry (EELS) [19] and parented cathodoluminescence (CL) [20]. For these methods the spatial resolution is, in first approximation, limited by the ability to focus an electron beam [19]. The latter can be as small as one nanometer. Recent successes regarding high-resolution mappings of nanoplasmonic resonances [19] demonstrate the strong capabilities of the EELS STEM (scanning transmission electron microscope) in plasmonics with spatial resolution down to 9 nm. However, the excitation mechanism of plasmons with fast electrons is significantly different from optical excitation and correlating the resulting EELS maps with the local density of optical states is still a debated issue [21-23]. Furthermore, direct investigations with polarized excitation are so far not possible with electron excitation based methods [21].

The main decay channel of a plasmon excitation corresponds to ohmic losses. In more details, plasmons decay first into single electron excited states, that is (electron, hole) pairs. For excitation energies below the metal work function, the excited electrons decay non-radiatively through electron-electron (on a $\sim 10 \mathrm{fs}$ time basis) and electron-phonon collisions (on a $\sim 1$ ps time basis) yielding ultimately to a temperature increase of the substrate. Detection of the heat produced by plasmonic decay can be conducted by photoacoustic effect. Discovered by A.G. Bell in 1880 [24], the photoacoustic effect measures the conversion of light into acoustic waves through absorption and subsequent thermal heating of the substrate. The application of a photoacoustic technique to investigate the non-radiative decay of a surface plasmon was first proposed by Inagaki [25], later followed by several international groups [26-28]. To date, the photoacoustic effect in connection with plasmonics is mainly used to reveal the thermal signatures accompanying the plasmon resonances of metallic particles used as imaging contrast agents [29].

A recent panorama of the real-space microscopic imaging techniques devoted to plasmonics is available [30].

\section{Principles of scanning tunneling luminescence}

The STM permits a well-controlled atomic-scale electrical addressing of metal nanostructures. This nanoscale tunnel junction can also behave as a highly localized source of electromagnetic radiation at frequency up to the quantum limit $\omega_{C}=e\left|V_{B I A S}\right| / \hbar$, where $V_{B I A S}$ is the sample bias. For biases of $\sim 1 \mathrm{~V}$ and above this source has frequencies up to the optical domain, and can thus probe electronic and photonic properties with unparalleled spatial resolution. After its first observation with relatively high quantum efficiencies [31] STM-induced light emission, also named scanning tunneling luminescence (STL), was shown to be suitable for imaging with subnanometer resolution [32]. Despite the low intensity of the emission, generally detected through photon-counting techniques, the optical nature of the detection permitted local spectroscopic studies of rough metal surfaces [33,34] or single silver clusters [35].

To get insight into the mechanism of STL, let us consider the theoretical rate of spontaneous radiative emission $\Gamma_{\text {rad }}$ as derived from Fermi golden rule in the dipole approximation [36]:

$$
\Gamma_{\mathrm{rad}}=\frac{2 \pi}{\hbar^{2}} \mu_{i f}^{2} E_{Z}(\mathbf{M})^{2} \rho\left(\omega=\omega_{i f}\right)
$$

where $\mu_{i f}$ is the matrix element of the electric dipole moment operator for the considered transition $i \rightarrow f, \rho\left(\omega=\omega_{i f}\right)$ is the spectral density of electromagnetic modes taken at the transition frequency $\omega=\omega_{i f}$ and $E_{Z}$ (M) is the local zero-point rms electric field at the location $\mathbf{M}$ of the quantum system. Although $E_{Z}(\mathbf{M})$ may have fast spatial variations, it is in general legitimate to consider $\mu_{i f}$ as localized at point $\mathbf{M}$ [37], the former being estimated to have an extension of the order of $5 \mathrm{~nm}$ [38]. The rate of emission thus depends on two factors: a 'source' part which accounts for the electronic structure in the form of a dipole moment operator and a 'receptor' part which accounts for the local and spectral density of the excited electromagnetic eigenmodes, gap plasmon modes in our specific case. The spectral term of the latter, $\rho\left(\omega=\omega_{i f}\right)$, 


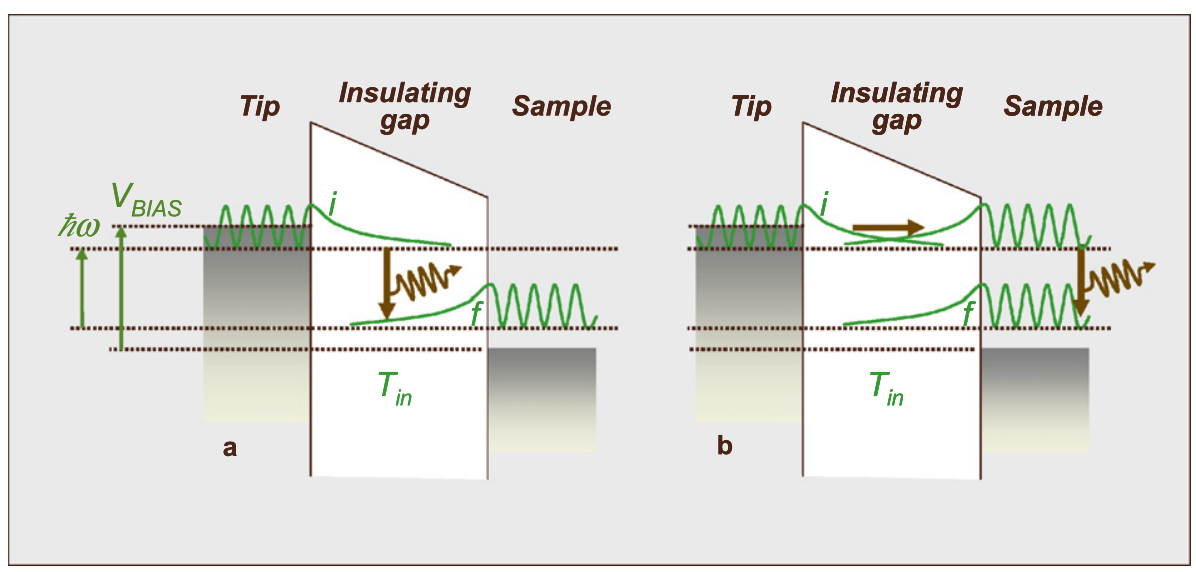

Fig. 1. Energy scheme of the two possible mechanisms for luminescence in an STM junction: (a) one-step emission through spontaneous emission (inelastic tunneling) from initial state $i$ to final state $f$, (b) two-step emission from elastic tunneling followed by radiative relaxation of hot electron.

represents the standard frequency-domain Purcell effect [39], whereas the spatial term $E_{Z}$ (M) represents enhancements through plasmon mode local confinement, discussed above. If empty-space plane waves are considered instead of plasmon modes the expression above leads to Einstein's $A_{\text {if }}$ coefficient [40], so that an enhancement factor, expressed as $\Gamma_{\text {rad }} / A_{\text {if }}$, can be evaluated in model situations from first principles using either classical $[41,42]$ or quantum [43,44] electrodynamics techniques.

Two main mechanisms can be involved as the origin of the source dipole moment $\mu_{i f}$ in STL, as sketched in Fig. 1. In both schemes, the tip and the substrate are brought close enough for electron tunneling, so that the evanescent parts of electron orbitals of the tip and the sample overlap in the insulating gap. The first mechanism (Fig. 1(a)) consists in a spontaneous emission from a filled state of the tip towards an empty state of the sample at lower energy. A nonzero transition dipole moment is made possible because of the overlap of their evanescent waves. This process corresponds to an inelastic tunneling from tip to sample, so that its efficiency can be more directly evaluated using transfer-Hamiltonian theory [45] which involves the ac momentum operator rather than the dipole moment [46]. The second mechanism (Fig. 1(b)) is a two-step process in which an elastic tunneling process generates an excited electron in the sample (hot electron) which subsequently relaxes radiatively [47]. However, under the tunneling conditions described in what follows, the former is the most effective mechanism [48].

The overall emission rate at frequency $\omega$ is the sum of the contributions by the available $(i, f)$ pairs verifying $\omega=$ $\left(E_{i}-E_{f}\right) / \hbar$. If both tip and sample electron density of states can be approximated as constants over the entire energy range thus defined, as well as their dipole moment matrix elements, the number of contributing $(i, f)$ pairs is proportional to the difference $V_{B I A S}-\left(E_{i}-E_{f}\right)[46,49]$. Hence, the source spectrum vanishes at the cut-off frequency $\omega_{C}=e\left|V_{B I A S}\right| / \hbar$ and increases progressively for decreasing frequencies. This wide spectrum is thus likely to largely overlap that of the plasmon modes, each mode at energy below the source cut-off being possibly excited by inelastic tunneling [50,51]. Furthermore, for a sample made in a uniform material, the electronic structure of the tip-sample junction is roughly unchanged when the tip scans the surface while maintaining a constant distance between the apex and the surface. Then, the source term in the tunneling-induced luminescence is constant and spatial contrast in emitted light intensity or spectrum can only be ascribed to plasmon-mode spatial profiles determined by the geometry of the nanostructured sample. Thus, STM-induced photon-emission map, acquired simultaneously with constant-current STM acquisition permits a high-resolution study of the electric-field distribution of the excited plasmon modes, provided the role of tip shape is carefully accounted for [52].

\section{Gap modes and tip-induced plasmon modes}

An important kind of plasmon modes is constituted by the so-called "gap modes" [53]. These modes appear when two metal nano-objects are approached one from another at a distance much smaller than their sizes and the wavelength of light. In particular, the geometry of the STM-junction, where a sharp metal tip - that is with curvature radius smaller than the wavelength of light - is brought at an electron-tunneling distance from a metallic surface, is highly favorable to the formation of gap modes [54]. These tip-induced plasmon modes are highly localized at the tip-surface junction yielding large local enhancements of the mode amplitude [55]. The lateral extension of the enhanced field region is typically of the order of $\sqrt{d R}$ where $d$ is the tip-sample distance and $R$ is the tip curvature [56], so that the confinement volume is of the order of $V=d^{2} R$. In the model of spherical tip apex and flat substrate made in the same metal, the resonance frequencies $\omega_{l}$ of these modes are given by the implicit relation:

$$
\frac{1}{\varepsilon\left(\omega_{l}\right)}=-\left(l+\frac{1}{2}\right) \sqrt{\frac{d}{2 R}}
$$


which involves the frequency-dependent dielectric constant of the metal $\varepsilon(\omega)$ and an integer mode index $l=0,1, \ldots$ For a metal described by a Drude model with plasmon frequency $\omega_{P}$ and in the case $d \ll R$ this equation gives explicitly:

$$
\omega_{l} \approx \omega_{P} \sqrt{\left(l+\frac{1}{2}\right) \sqrt{\frac{d}{2 R}}}
$$

A better understanding of the nature of these modes can be gained by considering the coupled plasmon modes of two metal spheres [57]. At large distance, i.e. weak-coupling, the lowest-frequency mode is formed by combination of the two single-sphere dipolar modes oscillating in-phase along their common axis. When $d$ is progressively reduced down to $d \ll R$, contributions from higher-order single-sphere modes become increasingly involved in this lowest-frequency mode of the coupled-sphere system, combined so as to increase the localization of the oscillations at the junction between the spheres. This corresponds to the formation of the lowest-order gap mode $(l=0)$ in the strongly coupled situation. Higher-order gap modes $(l>0)$ can be reproduced similarly by continuity from the weakly coupled limit to the strongly coupled situation, starting from higher-order (e.g. quadrupolar) weakly coupled modes of the two spheres.

Although a significant proportion of the mode energy is localized into the confinement volume $V=d^{2} R$ where field enhancements occur, spatially expanded contributions are preserved, including radiative contributions: SPPs and emptyspace far-field waves. The enhancement - in other words the quality factor $Q$ of the mode - is then controlled by the balance between the far-field radiation rate of this mode and electromagnetic losses in the tip and sample materials, these losses being described by the imaginary part of the dielectric constant. This feature permits the excitation (detection) of the gap modes using sources (detectors) located in the far-field regions. This property gave birth to a number of microscopy techniques exploiting various tip-enhanced optical processes, such as Tip-Enhanced Raman Scattering (TERS), a tip-localized version of Surface-Enhanced Raman Scattering (SERS) which is based on rough surfaces.

Although these techniques are usually based on a third-party light absorber, emitter, or optically nonlinear quantum system, e.g. an organic dye or a semiconductor quantum dot, nanostructured metal systems by themselves may also be the siege of luminescence phenomena with exploitable efficiencies after plasmon enhancements. For instance, photoluminescence has been observed on gold island films with increased efficiency compared to bulk Au and with spectral changes reflecting intrinsic modifications of the emission process [58]. Light emission from a single gold cluster [59] as well as a film of silver clusters excited by electron-tunneling current have been observed and interpreted in terms of electron-gas heating [60]. Efficient electro-luminescence from metal-oxide-metal devices involving one tunnel junction with rough interfaces has been observed and attributed to plasmon assisted inelastic electron tunneling [61].

\section{Experimental details of scanning tunneling luminescence}

Because of low quantum yields (typically much less than $10^{-4}$ ) and tunnel currents (typically in the nA range), the maximum rate of photon emission is usually of the order of a few $10^{5}$ emitted photons per second. Hence, the main experimental difficulty is the high efficiency required both for collection and detection. Photon-collection systems are often designed specifically, based on large-aperture aspheric lenses or mirrors together with low-loss either free-space or fiberbased optical routing of the signal to the detector located outside the UHV chamber (see e.g. [62]). The emitted light is detected with low dark-noise high sensitivity systems such as avalanche photodiode operating in a photon-counting mode, or cooled charge-coupled device arrays, the latter being mainly used in association with a spectral dispersion system for optical spectroscopy. Detectors with extended infrared sensitivity are preferred since they permit to study photon emission at lower applied biases, which are safer for the stability of surface quality.

Photon maps are acquired simultaneously with topographic maps by recording photon counts at each pixel for a fixed acquisition time. The STM operated in a constant-current mode. Since the bias is also constant, photon counts map directly the quantum efficiency of photon emission per tunnel electron. Defects in the regulation of the tunnel current to its setpoint, notably through a too-low response time of the feedback loop compared with scan speed, may cause instrumental artifacts in photon maps. A careful attention must thus be paid to the efficiency of the tunnel-current regulation. Ultimately, a systematic simultaneous acquisition of tunnel-current error signal can permit post-acquisition correction of the photon map so that it better reflects the ratio of inelastic to elastic tunneling.

Since the tip-induced formation of gap modes is mandatory to get measurable light-emission intensities, the choice of tip material strongly influences the STL signals [63]. Highest light-emission efficiencies are thus obtained with gold tips, despite their poor STM imaging capability. An often successful alternative is gold-covered tungsten tips.

Finally, it should be mentioned that STL can work at the liquid-solid interface. The immersion of the junction in liquid has been shown to affect the light-emission response both because of its influence on tip-to-sample elastic and inelastic electron-tunneling processes and because of a dielectric constant different from that of vacuum which alters the plasmon resonance frequencies [64].

\section{Applications of scanning tunneling luminescence to plasmonic-field mapping}

The first example we provide here is that of protrusions formed by the tip itself [65]. When voltage pulses are applied on an STM junction, atoms can be released from the tip and deposited on the surface. This process is especially effective 

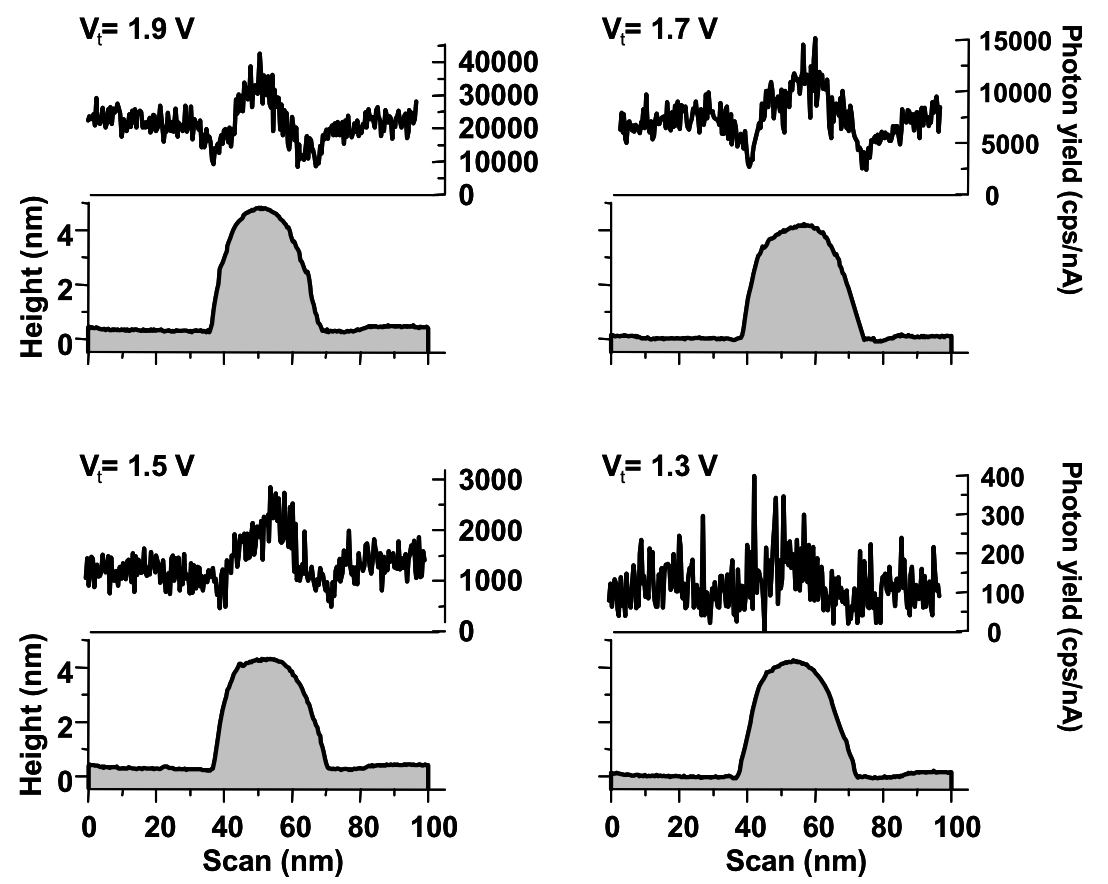

Fig. 2. Cross-sections of topography (lower curves with greyed area) and profiles of photon emission yields (upper curves) acquired simultaneously for a scan line crossing the middle of a tip-generated gold bump. The set-point is $I_{T}=1 \mathrm{nA}$ and the bias is $V_{B I A S}=1.9 \mathrm{~V}$ (upper left graph), $V_{B I A S}=1.7 \mathrm{~V}$ (upper right), $V_{B I A S}=1.5 \mathrm{~V}$ (lower left) and $V_{B I A S}=1.3 \mathrm{~V}$ (lower right). Adapted from Charra [65].

when gold tips are used and can be exploited in the more or less controlled formation of nanoscale bumps on gold (111) substrates [66]. This offers the opportunity to study the STM-induced photon-emission properties of such individual tipformed protrusions.

The STL photon map exhibits strong variations in luminescence quantum yields above such a bump as well as in its close vicinity. Superimposed sectional views of topography and photon maps are reproduced in Fig. 2, for different values of the bias. In all photon map profiles, the photon yields are nearly constant on the flat surface, except in an area located within $\sim 10 \mathrm{~nm}$ from the bump external limits, where the yield systematically decreases. This effect is especially visible for the largest biases where an angular point is clearly visible at the minimum of the photon rate. Accounting for the convolution with tip shape, topography profiles show that the minimum of photon emission corresponds to the sudden change of the location of the tunnel current, from the surface to the bump. Another clear feature of photon-emission profiles is an increase in efficiency near the maximum of the bump. This increase amounts to $\sim 50 \%$ of the flat terrace uniform emission, nearly independently of the bias.

Since the terrace and the bump are made of gold, the source term and the local dielectric constants can be considered as uniform. Hence, the origin of the observed contrasts in STL can only be ascribed to changes in the geometry of the junction, which influences the extension of the plasmon-mode confinement region. The change in photon rate observed when the tip approaches the bump while the topographic profile is still flat shows that the photon-emission process senses the presence of the bump at a larger distance than electron tunneling. This proves that the spatial extension of plasmon mode is much larger than that of the tunnel current. The decrease can be explained by an extension of the plasmon mode towards the bump where the tip-sample distance is reduced. As explained above, such an extension of the plasmon volume decreases the electron-plasmon coupling. This interpretation is further confirmed by the observation that the slope is reversed precisely at the point where the current flips towards the bump. Similarly, when the tip apex is located on the top of the bump, the plasmon mode is better localized due to substrate curvature which significantly increases this coupling.

Highly original plasmonic phenomena take place in dense assemblies of noble-metal nanoparticles or nanocrystals. Nanochemistry permits the fabrication of nearly perfect nanosized metal spheres. Such nanoparticles self-assemble into hexagonal monolayers identically replicated with a long-range order [67,68]. Such structures provide well-suited model systems to explore the original plasmonic properties emerging at dimensions for which STL mapping is the only technique offering the necessary resolution.

The example we provide here consists in self-assembled close-packed monolayers of $4 \mathrm{~nm}$ dodecanethiol-coated silver nanospheres [67]. The particles were deposited on $\mathrm{Au}(111)$ substrates grown on mica by applying one droplet of solution, and immediately reintroducing the sample into UHV. 

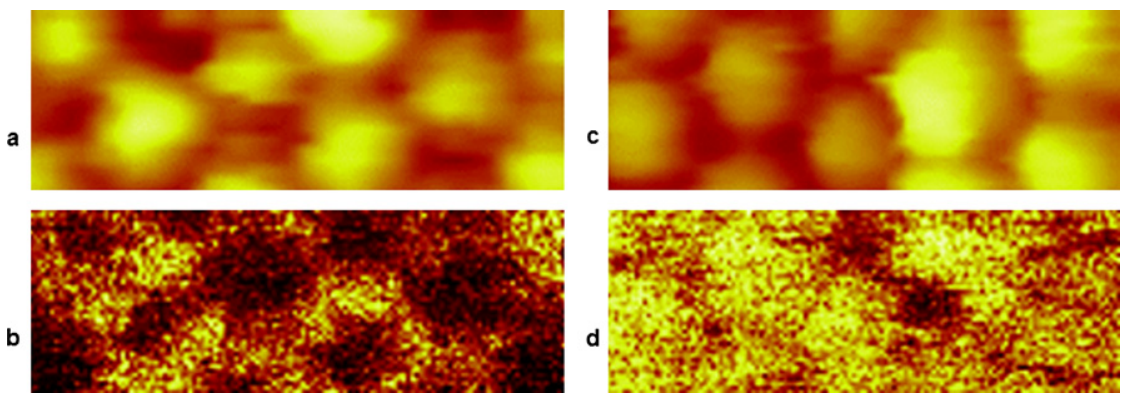

Fig. 3. High-resolution STM height images (upper images, a and c; image size: $26 \mathrm{~nm} \times 10 \mathrm{~nm}$; set-point $I_{T}=3.5 \mathrm{nA}$ ) and corresponding maps of the simultaneously acquired STL (lower images, b and d) for two different biases: $V_{B I A S}=2.1 \mathrm{~V}$ (left, a and b), $V_{B I A S}=2.5 \mathrm{~V}$ (right, c and d). Adapted from Silly et al. [69].

A topographic STM image of a monolayer of $4.3 \mathrm{~nm}$ diameter silver nanospheres is shown in Fig. 3. The spontaneous hexagonal arrangement of the highly homogeneous particles is visible [70]. Other kinds of supra-crystalline phases have been observed, with important consequences on electron-transport properties [71].

A close-up onto a self-assembled domain is also shown. The STM height images and corresponding STL photon maps were recorded simultaneously. Two topography/luminescence pairs were recorded on the same area successively using different biases $V_{B I A S}=2.1 \mathrm{~V}$ (left images) and $V_{B I A S}=2.5 \mathrm{~V}$ (right image). At the lower bias, the photon-emission efficiency reaches a maximum when the tip is located exactly above the junction between two neighboring particles, whereas photon emission drops below the detection limit at the top of a particle. In contrast, at higher biases, the maximum of photon emission is detected on the top of each particle and a minimum at the junction between particles. The emission rate measured at this minimum at $V_{B I A S}=2.5 \mathrm{~V}$ is still larger than that measured at the same location at $V_{B I A S}=2.1 \mathrm{~V}$, so that there is still no decrease in emission efficiency for increasing bias.

As discussed above, such contrasts in STL correlated with the geometry must be attributed to plasmon effects, and reflect the spatial variations of their amplitudes when the tip is scanned. The lowest-energy unperturbed plasmon mode of an isolated silver sphere $(2.9 \mathrm{eV})$ being larger than the excitation biases applied in the above example (up to $2.5 \mathrm{~V}$ ), we are led to consider coupled, or collective, plasmon modes [72]. The photon map at lowest bias shows that, similarly with the simple two-sphere system [57], the amplitude of the optical field in the lowest-frequency modes is located in the region between the spheres. The expected in-plane polarization of these modes explains that no photon emission is observed when the tip is located over the top of a particle, since then their excitation is forbidden by symmetry. Modes at higher frequency involve single-particle oscillations polarized perpendicular to the plane (to the axis formed by the sphere centers in the 2-sphere model). Although the extended collective modes of the densely packed 2D self-assembled network are inherently different from those of the simple 2-sphere model, for small inter-particle distances, the lowest-frequency modes are highly localized at the junctions between spheres. Although the presence of the tip perturbs this scheme, the strength of tipsample electromagnetic interactions follows the local amplitude of the sample plasmon modes. When the tip is located over a particle, a standard tip-induced gap mode is formed between this particle and the tip, the coupling between particles becoming less important. These modes are excited at higher biases, which confirms their higher frequency compared with particle-junction modes. Hence, these photon maps give new insights into the microscopic structure of plasmonic modes in such self-assembled 2D nanoparticle assemblies. Their excitation energy as well as their $s / p$ polarization dependence permit the assignment of the various peaks observed in macroscopically measured spectra.

Another striking example of application of STL imaging for plasmonic investigations is given by the recent study by Myrach et al. [73] of the plasmon modes of nearly cube-shaped Ag particles grown on an MgO thin film. At liquid-nitrogen temperature, highly structured light-emission patterns were recorded with intra-particle resolution.

As for dense nanoparticle systems presented above, photon maps exhibit strong dependence on the applied bias (Fig. 4). However here, the low density of particles precludes any inter-particle couplings. The authors show that such a response can only result from the specific structure of the plasmon modes available for assisting the light-emission process at the each given applied voltage. More specifically, they show that the detected patterns at emission maxima can be explained in terms of in-plane (horizontal) and out-of-plane (vertical) plasmon modes of the Ag nano-cubes. These assignments are confirmed by the spectral measurements of the emitted light in each specific set of conditions, which correlates with edges and top-facet center emissions.

Again, like for coupled nanoparticles, the polarization of the modes changes from horizontal to vertical when varying the excitation bias. Although the excitation geometry, involving vertical electron tunneling and thus vertical transition dipole moments, seems hardly compatible with excitation of horizontally polarized modes, in both cases the 3D nature of the objects may favor this process.

The plasmon modes involved in the nanoparticle systems mentioned in the preceding examples are highly localized modes which can be understood in a quasi-static framework. However, as we noticed in the theoretical introduction of STL, the excited plasmon must present spatially expanded radiative contributions such as SPPs and empty-space far-field waves in order to be able to extract light energy up to the detector necessarily placed in the far-field zone. This has been 

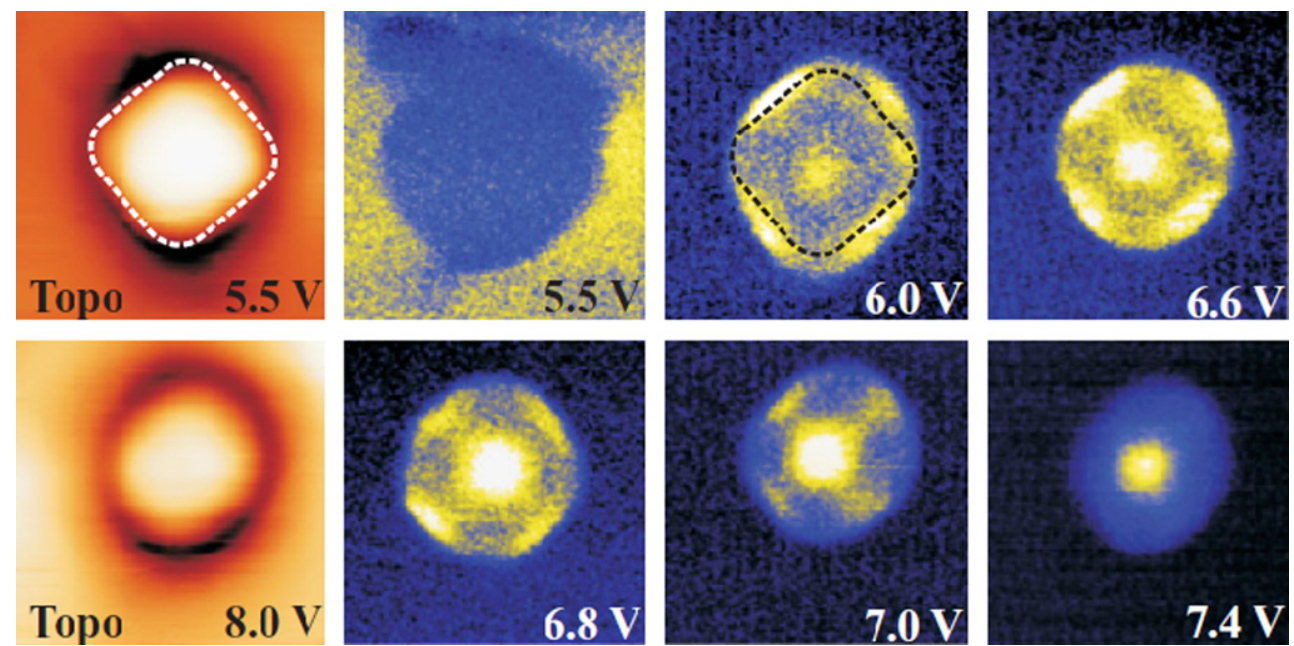

Fig. 4. STM topographic images and photon maps of an $\mathrm{Ag}$ particle on $\mathrm{MgO} / \mathrm{Mo}(001)$ taken as a function of sample bias $\left(I_{T}=1 \mathrm{nA}\right.$, $\left.8 \mathrm{~nm} \times 8 \mathrm{~nm}\right)$. The variations of luminescence distribution reflect the different in-plane and out-of-plane excited plasmon modes. Adapted from Myrach et al. [73].

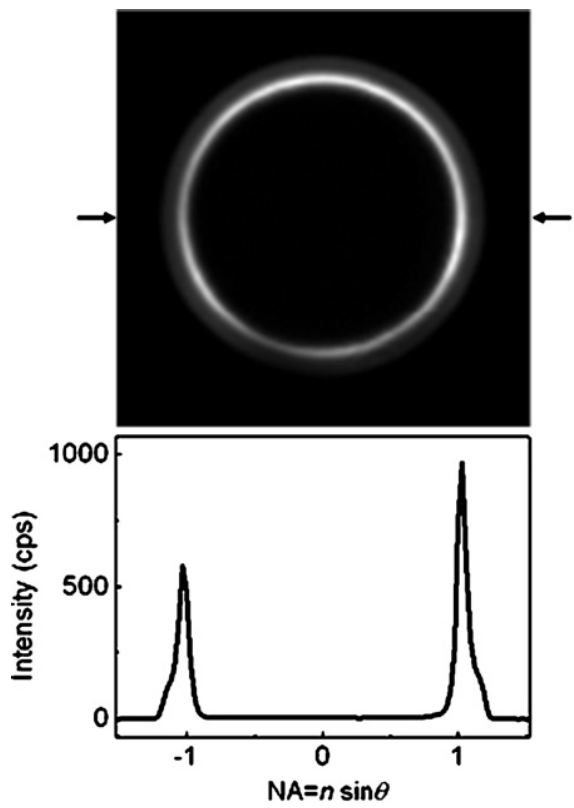

Fig. 5. STL collected through the rear face of the quartz substrate of an air/35 nm Au/quartz system $\left(I_{T}=5 \mathrm{nA}, V_{B I A S}=2.5 \mathrm{~V}\right)$. Image recorded for $60 \mathrm{~s}$ in the Fourier plane using an oil-immersion objective. The scale is expressed as a numerical aperture with the quartz refractive index $n=1.4585$ and the exit angle $\theta$. Adapted from Wang et al. [74].

demonstrated unambiguously recently by Wang et al. [74], who demonstrated the excitation of propagating SPPs on a thin gold film by inelastic tunneling. The map of the directions of the emerging light unambiguously shows that the majority of the detected photons transit as propagating SPPs as reproduced in Fig. 5. Actually, most of the luminescence is emitted at an angle close to the refractive limit, as expected for leakage radiation from propagating SPPs [75]. From SPP dispersion relation calculations, the authors then estimated that the most intense wavelength of STL should be in the range 650-800 nm.

\section{Surface plasmons and multiphoton electron emission}

While internal plasmon damping by electron photoemission opens a way to precise near-field investigations, practical implementations first encountered both physical and technical difficulties. Indeed, in the framework of a linear photoelectric effect, the decay of an SP wave implies a quantum energy greater than the metal work function [76]. For noble metals, plasmon energies correspond to near infrared/visible range, i.e. $380 \mathrm{~nm}(3.26 \mathrm{eV})-1400 \mathrm{~nm}(0.885 \mathrm{eV})$, while surface work functions amount to several $\mathrm{eV}$ (around $4.5 \mathrm{eV}$ for $\mathrm{Cu}, \mathrm{Ag}, \mathrm{Au}$ [77]). Consequently, plasmonic investigations conducted by direct linear electron photoemission are nearly impossible, except after artificial decreases of the metal work function by, 
for instance, alkali metal deposition. Indeed, the deposition of a few Å cesium film on silver or gold decreases significantly their work functions allowing the investigation of plasmon resonances by linear electron photoemission [78-80].

Beyond the linear photoelectric effect, electron photoemission can be strongly enhanced upon excitation of plasmons [ 81 , 82]. The advent of laser sources delivering ultrashort time pulses of light, i.e. within femtosecond (fs) to picosecond (ps) range, opened the field of nonlinear optics [83-86]. When an intense light pulse irradiates a metallic surface, the electron emission from the surface stems from the absorption of incident photons, the absorption of higher harmonic photons (SHG...), the increase in the electron temperature or the electron tunneling through the surface potential barrier (field emission) [87-89]. In the following, we will limit ourselves to the regime where the process of multiphoton absorption dominates at visible and near infrared wavelengths. Making use of the scale parameter $\gamma$ introduced by Keldysh, multiphoton processes dominate for the case $\gamma \gg 1$. For noble metals excited in the near infrared range, the latter inequality yields to low irradiances of the order of $\sim 100 \mathrm{MW} / \mathrm{cm}^{2}$. In this range, works dealing explicitly with the role played by plasmons on the photoemission process are of particular interest. Among pioneering examples [90-92], Stuckless and Moskovits early evidenced orders-of-magnitude enhancement of the two-photon photoemission process from rough silver films over those from smooth annealed films and ascribed it to "an increased local field strength near the surface upon resonant excitation of localized surface plasmons". Tsang, Srinivasan-Rao and Fischer gave further evidence of a surface-plasmon field enhanced multiphoton photoelectric emission from metal surface [93]. Advancing in time, similar observations surfaced regularly [89, 94-98].

According to standard description, a nonlinear $n$-photon photoelectron emission (nPPE) process has two possible physical origins. The first mechanism involves a direct vertical transition between occupied and unoccupied states via simultaneous absorption of $n$ photons (coherent absorption scheme). The second one considers an indirect transition described as a cascade photon absorption process. The direct transition mechanism implies virtual intermediate states and its cross-section decreases very rapidly with the number of absorbed photons. The indirect one involves real intermediate states above the Fermi level with the successive photon absorption events taking place within the lifetime of the excited states. For photon energies resonant with plasmon excitations, the strong amplitudes of the electric near field characterizing the plasmon translate into enhanced photoemission yield. To date the commonly accepted underlying mechanism for the increase of the photoemission yield observed close to a plasmon energy is an $n$-step cascade absorption process. The additional momentum transfer associated with indirect transition is presumably due to local defects, that is stacking faults, grain boundaries, impurities, ... [99].

\section{Photoemission electron microscopy (PEEM)}

The multiphoton photoemission investigations cited above made use of spatially integrating techniques and no considerations about real-space imaging have been taken. Considering electron as an imaging particle, a full set of emission electron microscopies exists from secondary electron microscopy (SEM) to thermionic electron microscopy (THEEM). Among this family, the photoemission electron microscopy (PEEM) offers a natural imaging technique likely to complement the spatially averaging plasmon enhanced multiphoton spectrometries. After decades of technical improvements, PEEM becomes an effective surface science tool during the 1980s [100]. For interested readers, a retrospective sketching the main steps of the evolution of the emission electron microscopies can be found [101].

Today PEEM is a widely used cathode lens electron microscopy, whose imaging principle consists in the collection of the electrons emitted by a surface (photons in, electrons out). It is a full field microscopy exploiting well-established electron optics (see Fig. 6) and no physical probe is involved (no moving part). Most frequently, the primary photoelectric process is linear in nature and is produced by sample excitation with UV light, X-ray laboratory sources or synchrotron radiation. For low photon energies, the image contrast corresponds to differences in work functions, electron density of states or topographical relief. At higher excitation energies, element-selective imaging is possible. Using UV light, a resolution down to $10-20 \mathrm{~nm}$ is routinely achieved. With the advent of aberration corrected instruments, image resolution approaches the ultimate resolution of electron emission microscopies, namely the inelastic mean free path of the emitted electrons [9].

Since the mid-1980s, PEEM has proven to be a powerful tool in surface science [102-105]. Among particularly successful application area, one can cite the investigation of chemical surface reaction (real time processes, spatiotemporal patterns) [106], surface diffusion [107], growth process [108,109] and magnetism [110].

\section{Photoemission microscopy, a tool for plasmonics}

First attempts to go beyond linear excitation appear during the 1980s. Massey et al. made use of the $266 \mathrm{~nm}$ fourth harmonic of an Nd:YAG laser to image guided waves in $\mathrm{LiNbO}_{3}$ by multiphoton absorption [111]. However, real-space imaging of nonlinear optical phenomena remains rare until the advent of reliable femtosecond laser sources. This stage is reached with the Ti:sapphire lasers which became commercially available in the mid-1990s.

Full application of PEEM in plasmonics is recent. Preliminary observations dealing with the spatial distribution of nonlinear effects in multiphoton photoemission experiments go back to 2001 [112]. However, physical measurements establishing clear connections between the nonlinear photoemission and the distribution of the optical near field associated to a plasmon resonance came a few years later. In 2005, Cinchetti et al. [113] clearly demonstrated the possibilities of the PEEM technique 


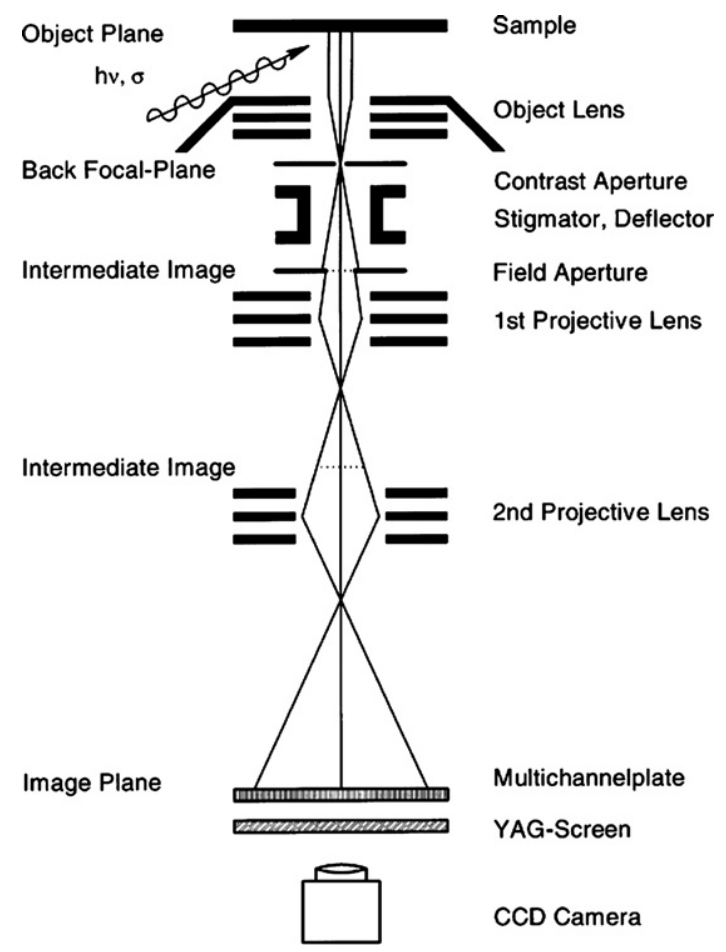

Fig. 6. Schematics of a photoemission electron microscope. Adapted from Swiech et al. [103].

as a tool for the investigation of the optical near field with the resolution of electron emission microscopy ( $25 \mathrm{~nm}$ ). Soon after, Kubo et al. [114,115] demonstrated what photoemission electron microscopy can bring to the plasmonic research area. Along with a tip free high-resolution near-field imaging technique, pump probe experiments were designed to precisely investigate the spatiotemporal aspects of the surface plasmon polariton propagation process. The active research groups currently contributing to the plasmonics through specific use of the nPPE-PEEM microscopy are still few [82,99,114-122], but advancing in time the interest of this technique is growing and new contributions appear regularly [123,124].

So far, $n$-photon photoemission (nPPE)-PEEM has proved especially worth in three kinds of plasmonic investigations, namely: (i) the sub-wavelength mapping of the near field of objects/structures of interest; (ii) the dynamics of SPPs (nanometer, femtosecond); and (iii) the manipulation of the near field.

\subsection{Sub-wavelength near-field mapping of plasmon resonances}

A first characteristic example dealing with high-resolution near-field mapping along with simple field manipulation is presented by Awada et al. [125]. The objects under interest are Au equilateral triangles of nanometer edge sides. Two localized surface plasmon resonances are studied, namely the in-plane dipolar and quadrupolar plasmon excitations. Experimental near-field maps are interpreted within the framework of a group theory description and finite difference time domain (FDTD) simulations. Starting with the $(\ell=1)$ dipolar mode of $E^{\prime}$ symmetry, its irreducible representation is of degeneracy 2, so the physical charge distributions at resonance correspond to linear combinations of a pair of two eigenvectors. Both eigenvectors obtained as symmetry adapted linear combinations (SALC) of $s$-type orbitals mimicking electrical charges attached to the prism corners are displayed in Fig. $7(a, b)$ together with the corresponding FDTD simulations (c, d). The SALC basis is constituted of the two linear combinations $(2,-1,-1)$ and $(0,-1,1)$, whose perpendicular polarizations are respectively aligned on the altitude and the edge of the considered equilateral triangle.

The nPPE-PEEM technique making use of a true optical excitation scheme (photon in, electron out), the polarization state of the excitation light can thus be easily controlled. When excited by an in-plane electric-field vector aligned along one of its edges, a nanoprism displays a two-spot near-field resonance reminiscent of the SALC $E^{\prime}(0,-1,1)$ state, see Fig. 8. Similarly, for excitation along triangle altitudes, dipolar resonances show one strong spot in qualitative agreement with the expected $E^{\prime}(2,-1,-1)$ SALC state. Similar conclusion conducted at shorter wavelength holds for the quadrupolar plasmon modes.

Beyond access to high-resolution sub-wavelength near-field maps, this work demonstrates the selective excitations, by lifting of degeneracy, of the different LSPR eigenmodes at the single object level. In particular, it paves the way to the active control of the near-field patterns of a resonant structure. This approach is general and applies to any nano-object, whatever its initial shape symmetry (triangle, bow-tie, rod, ...). 
(a)
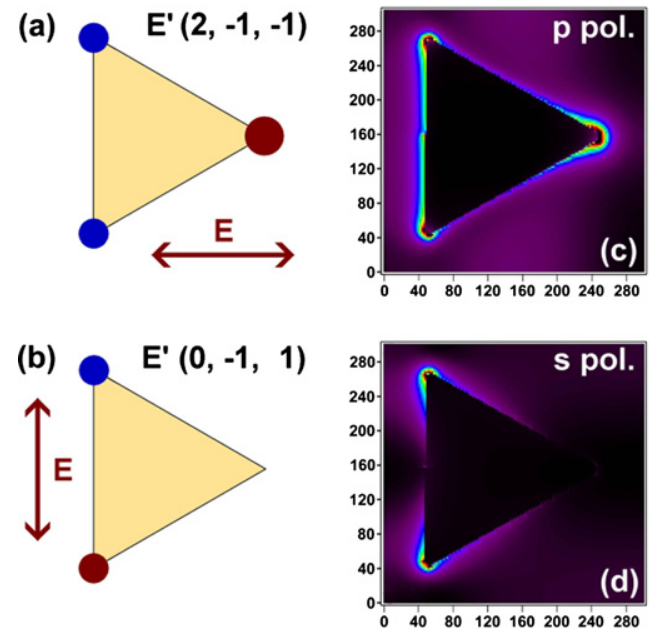

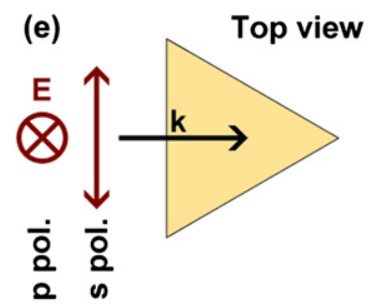

(f) Side view

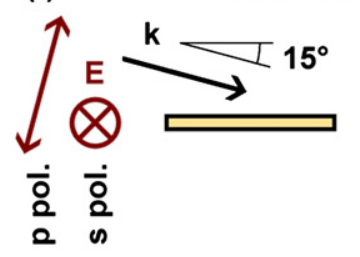

Fig. 7. Dipolar modes. (a, b) Symmetry adapted linear combinations (SALC) corresponding to the two dipolar eigenmodes of a solid triangle of $D_{3 h}$ symmetry. The charge patterns within the horizontal $\sigma_{h}$ plane exhibit two orthogonal polarization states. (c, d) Maps of the out-of-plane $\left(E_{z}\right)^{2}$ near field computed by FDTD simulations of the dipolar eigenmodes for $p \& s$ light polarizations. In-plane height of the triangle $200 \mathrm{~nm}$, excitation wavelength $800 \mathrm{~nm}$, light incidence $15^{\circ}$. (e, f) Top and side views of the illumination geometry. Adapted from Awada et al. [125].
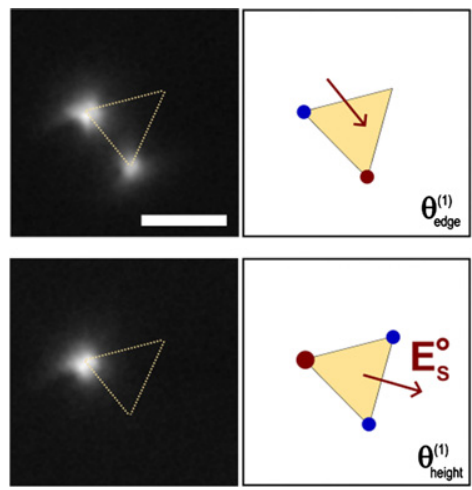
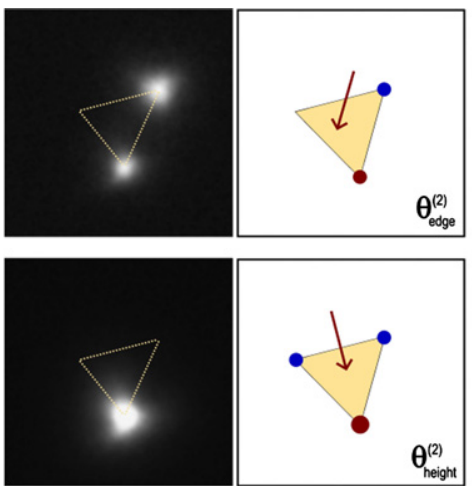
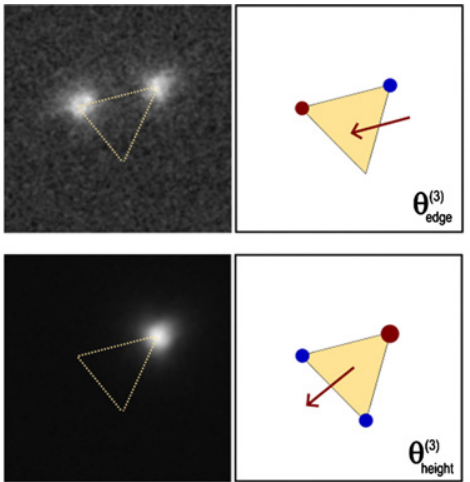

Fig. 8. Polarization dependence of the dipolar LSP resonances of a $200 \mathrm{~nm}$ in-plane height flat equilateral triangle. The top three images report the PEEM sequence recorded at the three successive polarization angles $\theta_{\text {edge }}$ corresponding to incident field polarized along triangle's edges. The bottom PEEM sequence corresponds to polarization angles $\theta_{\text {height }}$, i.e. incident field polarized along triangle's heights. For each polarization angle, a schematic of the right excited eigenmode is displayed. Photon wavelength $\lambda=800 \mathrm{~nm}$, beam incidence angle $\alpha=75 \pm 2^{\circ}$. White scale bar is $200 \mathrm{~nm}$. Adapted from Awada et al. [125].

A second example of high-resolution near-field mapping is given by Hrelescu et al. [119]. The authors have investigated the LSP resonances of nanostar shaped particles aimed at substrates for surface-enhanced Raman spectrometry (SERS) [126]. The nanoparticles consist in spherical metallic cores with 5 to 8 tips pointing outward and tip-to-tip diameters of 120$140 \mathrm{~nm}$, see characteristic LEEM and SEM pictures in Fig. 9(a, d). Under laser light excitation, these nanostars show bright spots of high photoemission yield located at the tips. This field distribution, ascertained via both in situ and post laserexcitation superpositions of morphology (LEEM, SEM) and near field (PEEM) signatures confirm theoretical models expecting localization of the optical excitation at the object's tips in the case of a parallel axis excitation [127]. Accordingly, the plasmonics hot spots of a single nanostar particle can be excited selectively by appropriately choosing wavelength and polarization of the excitation light, opening ways to simple and precise near-field spatial engineering at sub-wavelength scale.

\subsection{Dynamics of SPP field in space and time}

Regarding the dynamics of SPP waves, an illustrative example is proposed by Kubo et al. [114]. In this work, the authors monitored the evolution of an SPP wave packet at the vacuum/Ag interface by interferometric time-resolved (ITR) PEEM with $60 \mathrm{~nm}$ spatial resolution and 330 as ( 1 attosecond (as) $=1 \times 10^{-18} \mathrm{~s}$ ) as frame interval. The experiment setup showing the two-path interference scheme is recalled in Fig. 10(left). The external field excites an SPP wave at an Ag surface step edge acting as an additional source of momentum (surface discontinuity). This wave packet propagates away from the edge as a polarization wave $P_{\mathrm{SPP}}(x, t)$ along the vacuum/Ag interface (path 1 ). Simultaneously, the external field propagates along 

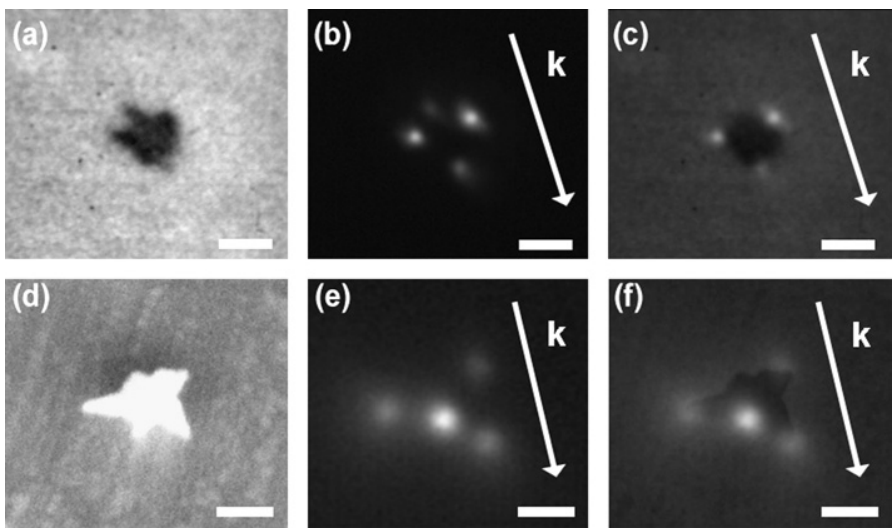

Fig. 9. High-resolution near-field mapping of individual colloidal nanostar shaped particles. (a) LEEM topography of an Au nanostar (nanostar I), (b) PEEM imaging of nanostar I under $730 \mathrm{~nm}$ laser excitation: composite image of two PEEM images obtained under different polarizations ( $p$ and $p+40^{\circ}$ ), (c) sum of (a) and (b), (d) SEM image of an Au nanostar (nanostar II), (e) PEEM imaging of nanostar II: composite image of three PEEM images obtained with $860 \mathrm{~nm}$ excitation and $p-42^{\circ}$-polarization, $780 \mathrm{~nm}$ excitation and $p$-polarization, and $860 \mathrm{~nm}$ excitation and $p+48^{\circ}$-polarization, respectively. (f) Sum of (d) and (e). For all PEEM images, the white arrow represents the in-plane projected $\boldsymbol{k}$-vector of the laser light (grazing incidence). The white scale bars represent $100 \mathrm{~nm}$. Adapted from Hrelescu et al. [119].
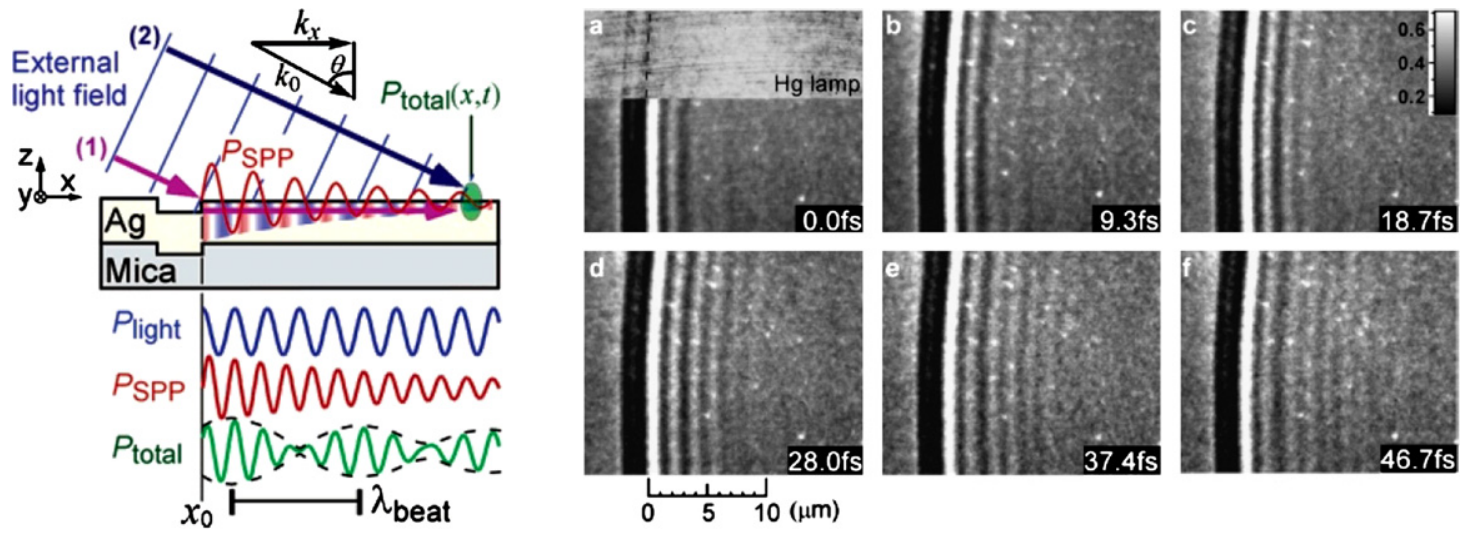

Fig. 10. Dynamics of SPP waves propagation investigated by pump probe experiment. (Left) Schematic of the experimental setup illustrating the two-path interference scheme between $P_{\text {SPP }}$ (path 1) and $P_{\text {light }}$ (path 2) imaged by ITR-PEEM through 2-photon absorption, (right) ITR-PEEM movie frames of the Ag film excited with phase-locked pump probe-pulse pairs. Bottom right numbers indicate the delay time (in fs) between pump probe pulses. Adapted from Kubo et al. [114].

path 2 and creates an additional local polarization component at the interface $P_{\text {light }}(x, t)$. Because of the phase velocity mismatch between both components, the total polarization excited by a single pulse exhibits an interference beating pattern. Excitation of the sample with phase correlated pulse pairs translates into interferences between the pump-pulse induced SPP wave $P_{\text {SPP }}$ and the probe-pulse induced $P_{\text {light }}$. Fig. 10(right) displays selected frames taken from an ITR-PEEM movie recorded with femtosecond laser incident from the left side imaging the SPP wave propagation.

Such direct investigations allow for the determination of quantitative characteristics of SPP waves such as wavelength, attenuation length, lifetime, dispersion curve, group and phase velocities... fixing numbers to be used in forthcoming technological applications. Recent illustrative examples can be found in [121,128-130].

\subsection{Near-field manipulation at will}

Full control of the near field of a resonant nanostructure on a spatial sub-wavelength length and time femtosecond scale has been demonstrated by M. Aeschlimann and co-workers [116,131,132]. Two control mechanisms have been mainly explored [133]: (i) a local sub-wavelength interference of the near-field object eigenmodes excited by two orthogonal polarization components of the incident light; (ii) a manipulation of the temporal evolution of the local near field.

A clear demonstration of the engineering possibilities of a near-field pattern is summarized in the following Fig. 11 [116]. Here, the near-field manipulation is achieved through a polarization shaping of the incident laser light pulse. A trigonal metallic nanostructure consisting of Ag disks $180 \mathrm{~nm}$ in diameter is illuminated by the light pulse of an fs-laser, its near-field pattern being investigated by photoemission electron microscopy. Subset (a) reflects a reference field distribution obtained for illumination with a standard unshaped $p$-polarized pulse. Subsets (c) and (d) display the experimental field pattern 


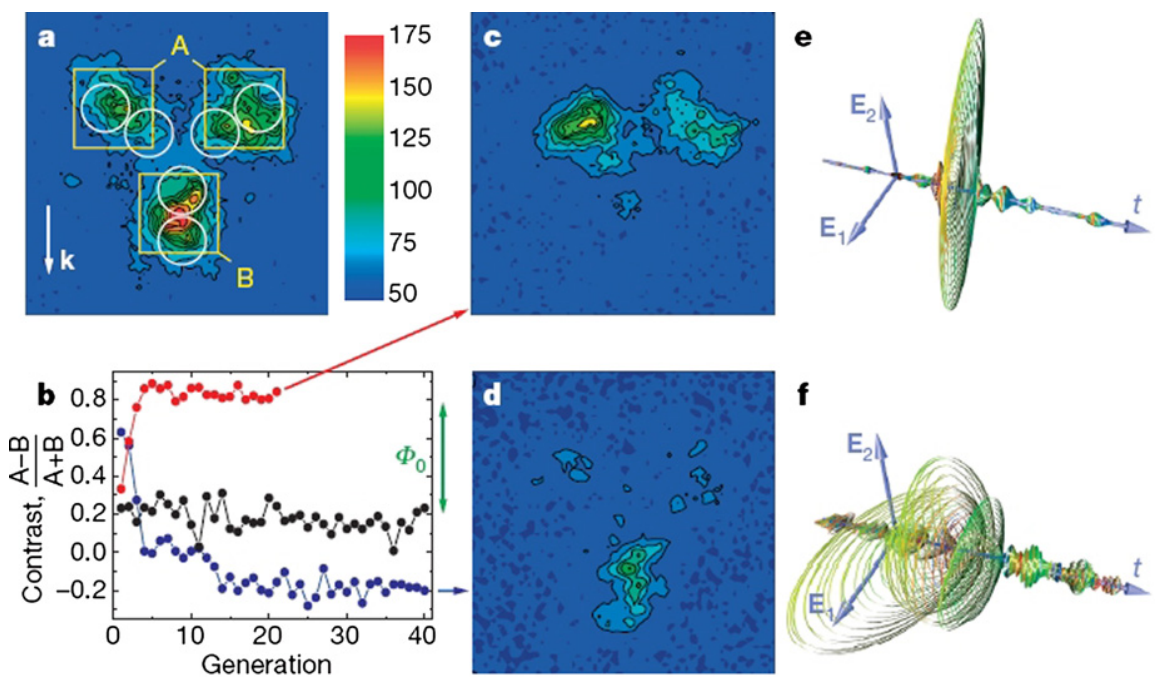

Fig. 11. Manipulation of near field through laser pulse shaping. (a) Experimental two-photon photoelectron distribution (1.13 $\mu \mathrm{m})^{2}$ obtained under unshaped p-polarized excitation at $790 \mathrm{~nm}$. The positions of Ag disks forming the star shaped nanostructure are marked as white circles, together with the regions A and B used for adaptative optimization. The white arrow represents the projected wave vector. (b) Adaptative optimization of the A/B photoemission ratio yields to increased (red) or decrease (blue) contrast between A and B regions as compared to reference pattern (a). (c) Experimental PEEM image after maximization using complex polarization-shaped pulses of the A/B ratio, (d) idem after A/B minimization. (e, f) Temporal evolutions of the vectorial electrical field $\mathbf{E}(t)$ defining the optimal shaped laser pulses for the maximization (e) and minimization (f) of the A/B ratio. Adapted from Aeschlimann et al. [116].

obtained using complex polarization-shaped pulses, whose electric-field temporal evolutions $\mathbf{E}(t)$ are shown in (e) and (f) respectively. Adaptative optimization of the laser pulse is carried out to maximize the photoemission contrast between regions $A$ and $B$ of the metallic structure (as defined in (a)).

In the above example, the optimal switching contrast is obtained through a close loop adaptive optimization of the polarization light pulse based on evolutionary algorithm, recent developments [131,132] propose open-loop control based on simple rule inferred from an analytical approach [134].

\section{Conclusions and perspectives}

Among the emerging techniques for real-space imaging of surface plasmons [30], STL offers unsurpassed resolutions. For instance, this technique permits the excitation of a luminescence of one individual metal nanoparticle out of a densely packed assembly in an adsorbed monolayer. Intra-particle features could even be observed, corresponding to resolutions below $1 \mathrm{~nm}$. The recent developments in time-resolved STL [135,136] may offer very exciting new perspectives in both domains. However, while offering a powerful local diagnostic tool in plasmonics, STL may request delicate deconvolution of the role of the tip itself.

To that respect PEEM microscopy appears as an alternative to scanned probe microscopies. In complement to the latter, photoemission microscopy probes the physics of plasmons from the metal side (electron reservoir). Beside its strength in near-field mapping, the use of an optical excitation scheme (photon in) ensures a strong connection to optical spectrometries, and opens wide experimental horizons in time domain investigations and near-field engineering [122].

\section{Acknowledgements}

We acknowledge financial support by the French National Agency (ANR) in the frame of its program in Nanosciences and Nanotechnologies (PEEMPlasmon project \# ANR-08-NANO-034) and by the Region Ile-de-France in the framework of C'Nano IdF, the nanoscience competence center of Paris Region, supported by CNRS, CEA, MESR and Region Ile-de-France (Plasmonics project).

\section{References}

[1] M. Faraday, Philosophical Transactions of the Royal Society 145 (1857).

[2] E.M. Purcell, Physical Review 69 (1946) 681.

[3] M. Moskovits, Reviews of Modern Physics 57 (1985) 783.

[4] P.E. Batson, Ultramicroscopy 9 (1982) 277.

[5] F. Hache, D. Ricard, C. Flytzanis, Journal of the Optical Society of America B - Optical Physics 3 (1986) 1647.

[6] T.W. Ebbesen, H.J. Lezec, H.F. Ghaemi, T. Thio, P.A. Wolff, Nature 391 (1998) 667.

[7] A. Lewis, M. Isaacson, A. Harootunian, A. Muray, Ultramicroscopy 13 (1984) 227. 
[8] D.W. Pohl, W. Denk, M. Lanz, Applied Physics Letters 44 (1984) 651.

[9] R.M. Tromp, Ultramicroscopy 111 (2011) 273.

[10] R.W. Wood, Philosophical Magazine 4 (1902) 396.

[11] A. Otto, Physica Status Solidi 26 (1968) K99.

[12] E. Kretschmann, H. Raether, Zeitschrift für Naturforschung, Teil A - Astrophysik, Physik und Physikalische Chemie 23 (1968) 2135.

[13] A. Garcia-Etxarri, I. Romero, F.J.G. de Abajo, R. Hillenbrand, J. Aizpurua, Physical Review B 79 (2009) 5.

[14] A.V. Zayats, I.I. Smolyaninov, Journal of Optics A - Pure and Applied Optics 5 (2003) S16.

[15] P. Ghenuche, S. Cherukulappurath, T.H. Taminiau, N.F. van Hulst, R. Quidant, Physical Review Letters 101 (2008) 4.

[16] M. Sandtke, L. Kuipers, Nature Photonics 1 (2007) 573.

[17] R.M. Bakker, A. Boltasseva, Z.T. Liu, R.H. Pedersen, S. Gresillon, A.V. Kildishev, V.P. Drachev, V.M. Shalaev, Optics Express 15 (2007) 13682.

[18] M. Rang, A.C. Jones, F. Zhou, Z.Y. Li, B.J. Wiley, Y.N. Xia, M.B. Raschke, Nano Letters 8 (2008) 3357.

[19] J. Nelayah, M. Kociak, O. Stephan, F.J.G. de Abajo, M. Tence, L. Henrard, D. Taverna, I. Pastoriza-Santos, L.M. Liz-Marzan, C. Colliex, Nature Physics 3 (2007) 348.

[20] E.J.R. Vesseur, R.D. Waele, M. Kuttge, A. Polman, Nano Letters 7 (2007) 2843.

[21] A.L. Koh, K. Bao, I. Khan, W.E. Smith, G. Kothleitner, P. Nordlander, S.A. Maier, D.W. McComb, ACS Nano 3 (2009) 3015.

[22] U. Hohenester, H. Ditlbacher, J.R. Krenn, Physical Review Letters 103 (2009) 4.

[23] F.J.G. de Abajo, M. Kociak, Physical Review Letters 100 (2008) 4.

[24] A.G. Bell, American Journal of Sciences XX (1880) 305.

[25] T. Inagaki, Y. Nakagawa, E.T. Arakawa, D.J. Aas, Physical Review B 26 (1982) 6421.

[26] B. Rothenhausler, J. Rabe, P. Korpiun, W. Knoll, Surface Science 137 (1984) 373.

[27] S. Negm, H. Talaat, Journal of Physics - Condensed Matter 1 (1989) 10201.

[28] R.J. Matelon, D.M. Newman, M.L. Wears, Review of Scientific Instruments 75 (2004) 2560.

[29] L. Tong, Q.S. Wei, A. Wei, J.X. Cheng, Photochemistry and Photobiology 85 (2009) 21.

[30] R. Vogelgesang, A. Dmitriev, Analyst 135 (2010) 1175.

[31] J.K. Gimzewski, J.K. Sass, R.R. Schlitter, J. Schott, Europhysics Letters 8 (1989) 435.

[32] R. Berndt, R. Gaisch, J.K. Gimzewski, B. Reihl, R.R. Schlittler, W.D. Schneider, M. Tschudy, Science 262 (1993) 1425.

[33] M.M.J. Bischoff, M. van der Wielen, H. van Kempen, Surface Science 400 (1998) 127.

[34] K. Ito, S. Ohyama, Y. Uehara, S. Ushioda, Surface Science 324 (1995) 282.

[35] P. Dumas, C. Syrykh, V. Makarenko, F. Salvan, Europhysics Letters 40 (1997) 447.

[36] R. Loudon, The Quantum Theory of Light, Oxford University Press, Oxford, 2003.

[37] K. Perronet, L. Barbier, F. Charra, Physical Review B 70 (2004) 201405(R).

[38] R. Berndt, R. Gaisch, W.D. Schneider, J.K. Gimzewski, B. Reihl, R.R. Schlittler, M. Tschudy, Physical Review Letters 74 (1995) 102.

[39] E.M. Purcell, Physical Review 69 (1946) 681.

[40] P. Meystre, M.I. Sargent, Elements of Quantum Optics, Springer, Berlin, 2007.

[41] R. Carminati, J.J. Greffet, C. Henkel, J.M. Vigoureux, Optics Communications 261 (2006) 368.

[42] M.R. Philpott, Journal of Chemical Physics 62 (1975) 1812.

[43] S.C. Ching, H.M. Lai, K. Young, Journal of the Optical Society of America B - Optical Physics 4 (1987) 2004.

[44] G. Sun, J.B. Khurgin, R.A. Soref, Applied Physics Letters 94 (2009) 101103.

[45] D. Hone, B. Muhlschlegel, D.J. Scalapino, Applied Physics Letters 33 (1978) 203.

[46] L.C. Davis, Physical Review B 16 (1977) 2482.

[47] J.R. Kirtley, T.N. Theis, J.C. Tsang, D.J. Dimaria, Physical Review B 27 (1983) 4601.

[48] B.N.J. Persson, A. Baratoff, Physical Review Letters 68 (1992) 3224.

[49] R.W. Rendell, D.J. Scalapino, B. Muhlschlegel, Physical Review Letters 41 (1978) 1746.

[50] P. Johansson, R. Monreal, P. Apell, Physical Review B 42 (1990) 9210.

[51] Y. Uehara, Y. Kimura, S. Ushioda, K. Takeuchi, Japanese Journal of Applied Physics, Part 1 - Regular Papers, Short Notes \& Review Papers 31 (1992) 2465.

[52] J. Aizpurua, S.P. Apell, R. Berndt, Physical Review B 62 (2000) 2065.

[53] H.X. Xu, J. Aizpurua, M. Kall, P. Apell, Physical Review E 62 (2000) 4318.

[54] A.G. Malshukov, Physics Reports - Review Section of Physics Letters 194 (1990) 343.

[55] P. Andre, F. Charra, M. Pileni, Journal of Applied Physics 91 (2002) 3028.

[56] R.W. Rendell, D.J. Scalapino, B. Muhlschlegel, Physical Review Letters 41 (1978) 1746.

[57] M. Schmeits, L. Dambly, Physical Review B 44 (1991) 12706.

[58] L. Khriachtchev, L. Heikkila, T. Kuusela, Applied Physics Letters 78 (2001) 1994.

[59] J.I. Gonzalez, T.H. Lee, M.D. Barnes, Y. Antoku, R.M. Dickson, Physical Review Letters 93 (2004) 147402.

[60] S.A. Nepijko, R.D. Fedorovich, L.V. Viduta, D.N. Ievlev, W. Schulze, Annalen der Physik 9 (2000) 125.

[61] J. Lambe, S.L. McCarthy, Physical Review Letters 37 (1976) 923.

[62] K. Kuhnke, A. Kabakchiev, W. Stiepany, F. Zinser, R. Vogelgesang, K. Kern, Review of Scientific Instruments 81 (2010) 113102.

[63] K. Kusova, F. Charra, G. Schull, I. Pelant, Surface Science 602 (2008) 345.

[64] K. Perronet, F. Charra, Physical Review B 67 (2003) 153402.

[65] F. Charra, in: M.-P. Pileni (Ed.), Nanocrystals Forming Mesoscopic Structures, Wiley-VCH Verlag GmbH \& Co. KGaA, Weinheim, Germany, 2005, p. 231.

[66] H.J. Mamin, P.H. Guethner, D. Rugar, Physical Review Letters 65 (1990) 2418.

[67] A. Taleb, C. Petit, M.P. Pileni, Chemistry of Materials 9 (1997) 950.

[68] P.J. Durston, J. Schmidt, R.E. Palmer, J.P. Wilcoxon, Applied Physics Letters 71 (1997) 2940.

[69] F. Silly, A.O. Gusev, A. Taleb, F. Charra, M.P. Pileni, Physical Review Letters 84 (2000) 5840.

[70] A.O. Gusev, A. Taleb, F. Silly, F. Charra, M.P. Pileni, Advanced Materials 12 (2000) 663.

[71] A. Taleb, F. Silly, A.O. Gusev, F. Charra, M.P. Pileni, Advanced Materials 12 (2000) 1583.

[72] A. Taleb, V. Russier, A. Courty, M.P. Pileni, Physical Review B 59 (1999) 13350.

[73] P. Myrach, N. Nilius, H.J. Freund, Physical Review B 83 (2011) 35416.

[74] T. Wang, E. Boer-Duchemin, Y. Zhang, G. Comtet, G. Dujardin, Nanotechnology 22 (2011) 175201.

[75] A. Bouhelier, T. Huser, H. Tamaru, H.J. Guntherodt, D.W. Pohl, F.I. Baida, D. Van Labeke, Physical Review B 63 (2001) 155404.

[76] T.A. Callcott, E.T. Arakawa, Physical Review B 11 (1975) 2750.

[77] H.L. Skriver, N.M. Rosengaard, Physical Review B 46 (1992) 7157.

[78] J. Bosenberg, Physics Letters A 37 (1971) 439.

[79] F. Sabary, J.C. Dudek, Vacuum 41 (1990) 476. 
[80] E.L. Nolle, M.Y. Schelev, Technical Physics 50 (2005) 1528

[81] P. Monchicourt, M. Raynaud, H. Saringar, J. Kupersztych, Journal of Physics - Condensed Matter 9 (1997) 5765.

[82] M. Munzinger, C. Wiemann, M. Rohmer, L. Guo, M. Aeschlimann, M. Bauer, New Journal of Physics 7 (2005) 68.

[83] S.I. Anisimov, V.A. Benderskii, G. Farkas, Soviet Physics - Uspekhi 20 (1977) 467.

[84] J.H. Bechtel, W.L. Smith, N. Bloembergen, Physical Review B 15 (1977) 4557

[85] R. Yen, J.M. Liu, N. Bloembergen, T.K. Yee, J.G. Fujimoto, M.M. Salour, Applied Physics Letters 40 (1982) 185.

[86] R.T. Williams, T.R. Royt, J.C. Rife, J.P. Long, M.N. Kabler, Journal of Vacuum Science \& Technology 21 (1982) 509.

[87] A. Gloskovskii, D. Valdaitsev, S.A. Nepijko, G. Schonhense, B. Rethfeld, Surface Science 601 (2007) 4706.

[88] P. Dombi, Advances in Imaging and Electron Physics, vol. 158, Elsevier Academic Press Inc., San Diego, 2009, p. 1.

[89] W. Pfeiffer, C. Kennerknecht, M. Merschdorf, Applied Physics A - Materials Science \& Processing 78 (2004) 1011.

[90] J.G. Endriz, W.E. Spicer, Physical Review Letters 27 (1971) 570.

[91] H.W. Rudolf, W. Steinmann, Physics Letters A 61 (1977) 471.

[92] U. Even, K.A. Holcomb, C.W. Snyder, P.R. Antoniewicz, J.C. Thompson, Surface Science 165 (1986) L35.

[93] T. Tsang, T. Srinivasanrao, J. Fischer, Physical Review B 43 (1991) 8870.

[94] N. Aeschlimann, C.A. Schmuttenmaer, H.E. Elsayed-Ali, R.J.D. Miller, J. Cao, Y. Gao, D.A. Mantell, Journal of Chemical Physics 102 (1995) 8606.

[95] J. Lehmann, M. Merschdorf, W. Pfeiffer, A. Thon, S. Voll, G. Gerber, Physical Review Letters 85 (2000) 2921.

[96] J. Kupersztych, P. Monchicourt, M. Raynaud, Physical Review Letters 86 (2001) 5180

[97] M. Maillard, P. Monchicourt, M.P. Pileni, Chemical Physics Letters 380 (2003) 704.

[98] G. Banfi, G. Ferrini, M. Peloi, F. Parmigiani, Physical Review B - Condensed Matter and Materials Physics 67 (2003) 10.

[99] C. Wiemann, D. Bayer, M. Rohmer, M. Aeschlimann, M. Bauer, Surface Science 601 (2007) 4714.

[100] W. Driesel, H. Bethge, in: Proc. Conf. on Energy-Pulse Modification of Semiconductors and Related Materials II, 1985.

[101] E. Bauer, Journal of Physics - Condensed Matter 21 (2009) 10.

[102] E. Bauer, M. Mundschau, W. Swiech, W. Telieps, Ultramicroscopy 31 (1989) 49.

[103] W. Swiech, G.H. Fecher, C. Ziethen, O. Schmidt, G. Schonhense, K. Grzelakowski, C.M. Schneider, R. Fromter, H.P. Oepen, J. Kirschner, Journal of Electron Spectroscopy and Related Phenomena 84 (1997) 171.

[104] S. Gunther, B. Kaulich, L. Gregoratti, M. Kiskinova, Progress in Surface Science 70 (2002) 187.

[105] A. Locatelli, E. Bauer, Journal of Physics - Condensed Matter 20 (2008) 22.

[106] S. Nettesheim, A. Vonoertzen, H.H. Rotermund, G. Ertl, Journal of Chemical Physics 98 (1993) 9977.

[107] H.H. Rotermund, S. Nettesheim, A. Vonoertzen, G. Ertl, Surface Science 275 (1992) L645.

[108] F. Heringdorf, M.C. Reuter, R.M. Tromp, Nature 412 (2001) 517.

[109] R. Gerlach, T. Maroutian, L. Douillard, D. Martinotti, H.J. Ernst, Surface Science 480 (2001) 97.

[110] G. Schonhense, Journal of Physics - Condensed Matter 11 (1999) 9517.

[111] G.A. Massey, M.D. Jones, J.C. Johnson, IEEE Journal of Quantum Electronics 17 (1981) 1035.

[112] O. Schmidt, G.H. Fecher, Y. Hwu, G. Schonhense, Surface Science 482 (2001) 687.

[113] M. Cinchetti, A. Gloskovskii, S.A. Nepjiko, G. Schonhense, H. Rochholz, M. Kreiter, Physical Review Letters 95 (2005) 47601.

[114] A. Kubo, K. Onda, H. Petek, Z.J. Sun, Y.S. Jung, H.K. Kim, Nano Letters 5 (2005) 1123.

[115] A. Kubo, Y.S. Jung, H.K. Kim, H. Petek, Journal of Physics B - Atomic, Molecular and Optical Physics 40 (2007) S259.

[116] M. Aeschlimann, M. Bauer, D. Bayer, T. Brixner, F.J. de Abajo, W. Pfeiffer, M. Rohmer, C. Spindler, F. Steeb, Nature 446 (2007) 301.

[117] L.I. Chelaru, M. Horn-von Hoegen, D. Thien, F.J.M. zu Heringdorf, Physical Review B 73 (2006) 5.

[118] L.I. Chelaru, F. Heringdorf, Surface Science 601 (2007) 4541.

[119] C. Hrelescu, T.K. Sau, A.L. Rogach, F. Jackel, G. Laurent, L. Douillard, F. Charra, Nano Letters 11 (2011) 402.

[120] L. Douillard, F. Charra, C. Fiorini, P.M. Adam, R. Bachelot, S. Kostcheev, G. Lerondel, M.L. de la Chapelle, P. Royer, Journal of Applied Physics 101 (2007) 83518.

[121] L. Douillard, F. Charra, Z. Korczak, R. Bachelot, S. Kostcheev, G. Lerondel, P.M. Adam, P. Royer, Nano Letters 8 (2008) 935.

[122] M.I. Stockman, M.F. Kling, U. Kleineberg, F. Krausz, Nature Photonics 1 (2007) 539.

[123] S.J. Peppernick, A.G. Joly, K.M. Beck, W.P. Hess, Journal of Chemical Physics 134 (2011) 7.

[124] R.C. Word, T. Dornan, R. Konenkamp, Applied Physics Letters 96 (2010) 3.

[125] C. Awada, T. Popescu, L. Douillard, F. Charra, A. Perron, H. Yockell-Lelievre, A.L. Baudrion, P.M. Adam, R. Bachelot, Journal of Physical Chemistry C 116 (2012) 14591

[126] C. Hrelescu, T.K. Sau, A.L. Rogach, F. Jackel, J. Feldmann, Applied Physics Letters 94 (2009) 3.

[127] F. Hao, C.L. Nehl, J.H. Hafner, P. Nordlander, Nano Letters 7 (2007) 729.

[128] H.S. Lee, C. Awada, S. Boutami, F. Charra, L. Douillard, R.E. de Lamaestre, Optics Express 20 (2012) 8974.

[129] L.X. Zhang, A. Kubo, L.M. Wang, H. Petek, T. Seideman, Physical Review B 84 (2011) 245442.

[130] C. Lemke, T. Leissner, S. Jauernik, A. Klick, J. Fiutowski, J. Kjelstrup-Hansen, H.G. Rubahn, M. Bauer, Optics Express 20 (2012) 12877.

[131] P. Melchior, D. Bayer, C. Schneider, A. Fischer, M. Rohmer, W. Pfeiffer, M. Aeschlimann, Physical Review B 83 (2011) 235407.

[132] M. Aeschlimann, M. Bauer, D. Bayer, T. Brixner, S. Cunovic, A. Fischer, P. Melchior, W. Pfeiffer, M. Rohmer, C. Schneider, C. Struber, P. Tuchscherer, D.V. Voronine, New Journal of Physics 14 (2012) 33030.

[133] T. Brixner, F.J.G. de Abajo, J. Schneider, C. Spindler, W. Pfeiffer, Physical Review B 73 (2006) 125437.

[134] P. Tuchscherer, C. Rewitz, D.V. Voronine, F.J.G. de Abajo, W. Pfeiffer, T. Brixner, Optics Express 17 (2009) 14235.

[135] F. Silly, F. Charra, Applied Physics Letters 77 (2000) 3648.

[136] K. Perronet, G. Schull, P. Raimond, F. Charra, Europhysics Letters 74 (2006) 313. 